\title{
Courant Algebroids. A Short History ${ }^{\star}$
}

\author{
Yvette KOSMANN-SCHWARZBACH
}

Centre de Mathématiques Laurent Schwartz, École Polytechnique, F-91128 Palaiseau, France

E-mail: yks@math.polytechnique.fr

URL: http://www.math.polytechnique.fr/cmat/kosmann/

Received December 03, 2012, in final form February 14, 2013; Published online February 19, 2013 http://dx.doi.org/10.3842/SIGMA.2013.014

\begin{abstract}
The search for a geometric interpretation of the constrained brackets of Dirac led to the definition of the Courant bracket. The search for the right notion of a "double" for Lie bialgebroids led to the definition of Courant algebroids. We recount the emergence of these concepts.
\end{abstract}

Key words: Courant algebroid; Dorfman bracket; Lie algebroid; Lie bialgebroid; generalized geometry; Dirac structure; Loday algebra; Leibniz algebra; derived bracket

2010 Mathematics Subject Classification: 01A65; 53D17; 17B62; 17B66; 22A22; 53C15

I dedicate this little memoir to Peter Olver on the occasion of his 60th birthday, with friendship and in admiration.

In 1986, in the historical notes to his Applications of Lie Groups to Differential Equations [38], Peter Olver pointed out that the concept of a "Poisson structure" was already known to Sophus Lie, under the name "function group," a century before André Lichnerowicz formally defined Poisson manifolds in 1977 [25] $]^{1}$. Ten years before Lichnerowicz's article, and in a completely unrelated inquiry, Jean Pradines had defined the new concept of Lie algebroid as the infinitesimal counterpart of Ehresmann's differentiable groupoids, which are to-day called Lie groupoids [39]. Both the geometry of Lichnerowicz's Poisson manifolds and the Ehresmann theory of groupoids developed separately until the existence of a relationship between the two theories was revealed when several mathematicians and mathematical physicists independently "discovered" the Lie bracket of differential 1-forms on a Poisson manifold ${ }^{2}$. In 1987, when Alan Weinstein, with Alain Coste and Pierre Dazord, proved that the Lie algebroid of a symplectic groupoid is the cotangent bundle of the base manifold equipped with this bracket of 1-forms [2], it became clear that, for any Poisson manifold, there is a Lie algebroid structure on the cotangent bundle. In fact, there is one more important property. The tangent and cotangent bundles together constitute a Lie bialgebroid. In 1988, Weinstein introduced the concept of a Poisson groupoid [52], and, in a joint paper published in 1994, Kirill Mackenzie and Ping Xu defined the concept of a Lie bialgebroid as the infinitesimal counterpart of Poisson groupoids [36]. A year later, Kosmann-Schwarzbach proved that a pair of Lie algebroids in duality form a Lie bialgebroid if and only if they define a pair of differential Gerstenhaber algebras ${ }^{3}$, i.e., the Lie algebroid structure of one defines a differential which is a derivation of the Gerstenhaber bracket of the exterior algebra of the

${ }^{\star}$ This paper is a contribution to the Special Issue "Symmetries of Differential Equations: Frames, Invariants and Applications". The full collection is available at http://www.emis.de/journals/SIGMA/SDE2012.html

${ }^{1}$ See also Alan Weinstein's earlier historical note in Expositiones mathematicae [54]. For the development of Poisson geometry up to 1998, see Weinstein's survey [53]. For more information on the history of Poisson brackets and Poisson geometry, see several chapters in the forthcoming book [21].

${ }^{2}$ For a modern treatise on Lie groupoid and Lie algebroid theory, see [35]. For elements of the history of the bracket of 1-forms on Poisson manifolds, see, e.g., footnote 5 in my survey [19].

${ }^{3}$ Gerstenhaber algebras are also called Schouten algebras. In fact, they are an abstract version of the algebra of multivector fields on a manifold, equipped with the Schouten-Nijenhuis bracket. 
other Lie algebroid [16]. A Poisson structure on a manifold thus gives rise to the prototypical example of such a Lie bialgebroid, consisting of the pair of the tangent and cotangent bundles.

Lie bialgebroids were "just" a generalization of the Lie bialgebras that are the infinitesimal counterpart of the Poisson Lie groups. The question that therefore arose was to define the double of such an object, just as Vladimir Drinfeld had defined the double of a Lie bialgebra. His definition, when $\left(\mathfrak{g}, \mathfrak{g}^{*}\right)$ is a Lie bialgebra, of the unique Lie algebra structure on $\mathfrak{g} \oplus \mathfrak{g}^{*}$ which leaves the canonical symmetric bilinear form invariant, and restricts to the given Lie algebra structures on $\mathfrak{g}$ and $\mathfrak{g}^{*}$, is contained in his 3 -page 1983 paper $[8]^{4}$. The problem turned out to be more complicated than it seemed, and it eventually led to the definition of Courant algebroids by Zhang-Ju Liu, Alan Weinstein and Ping Xu in 1997 [29]. The question that was posed had, as a special case, the problem - which had been raised long before in Poisson geometry - of defining a bracket on the sections of the Whitney sum of the tangent and cotangent bundles of a Poisson manifold, combining the Lie bracket of vector fields and the above-mentioned bracket of 1 -forms ${ }^{5}$.

There was an even more "elementary" problem in differential geometry, how to define a bracket with reasonable properties on the direct sum of the vector space of vector fields and the vector space of differential 1-forms which restricts to the Lie bracket of vector fields and vanishes on differential forms. It was Theodore (Ted) Courant's achievement to define such a bracket in his thesis, published in 1990 [3]. Below I shall describe the motivation for Courant's work, coming from Dirac's theory of constraints, and the work that Irene Ya. Dorfman developed independently, in the context of the Hamiltonian structures, i.e., the infinite-dimensional Poisson structures, arising in field theory, in the late 1980's and until her untimely death ${ }^{6}$.

Dmitry Roytenberg advanced the general theory of Courant algebroids further by founding it on supermanifold theory in his thesis, completed in 1999, but which has remained unpublished [41], and in his subsequent work [40, 43, 44]. He adopted the "cotangent philosophy" of Kirill Mackenzie according to which, in particular, given a Lie algebroid $A$, the cotangent bundle $T^{*}(A) \approx T^{*}\left(A^{*}\right)$ is a more fundamental object than the bundle $A \oplus A^{*}$. He also made use of the supergeometry approach that he had learned from Theodore (Ted) Voronov.

This different, more categorical line of inquiry concerning the concept of a "double" for Lie bialgebroids was explained by Mackenzie in several publications, starting in 1992 [31, 32, 33] and expounded in his article, first a preprint on arXiv in 2006, submitted to Crelle's Journal in 2008, but finally published only in 2011 [34]. (Double Lie algebroids are the infinitesimal counterparts of double Lie groupoids, and Mackenzie's theory includes many constructions besides the doubles of Lie bialgebroids.) The question of the double of a Lie bialgebroid was also treated by Ted Voronov in [50], using a "super" approach and the notion of graded manifolds. In [51], he characterized double Lie algebroids in terms of supergeometry and he proved that "Roytenberg's and Mackenzie's pictures give the same notion of a double of a Lie bialgebroid."

The rich theory of the "generalized geometry" of $T M \oplus T^{*} M$ was developed, first by Nigel Hitchin in 2003 [12], then by Marco Gualtieri in his thesis submitted in 2003, available on arXiv in 2004, but published much later as [11], and it has become a domain of research in its own right ${ }^{7}$.

\footnotetext{
${ }^{4}$ Drinfeld does not use the word "double". The definition of a "Manin triple" is to be found in his landmark "Quantum groups" article which appeared in 1987 in the proceedings of the International Congress of Mathematicians (Berkeley, 1986). The actual lecture was delivered on very short notice by Pierre Cartier because Drinfeld was not allowed to travel to the United States for the conference.

${ }^{5}$ I remember discussing this problem with Franco Magri circa 1990, and not finding a satisfying answer.

${ }^{6}$ Irene Dorfman (born 1948), one of the leading experts on integrable systems, died in Moscow in 1994. For her life and works, see the obituary by Oleg I. Mokhov, Sergei P. Novikov and Andrei K. Pogrebkov [37].

${ }^{7}$ The vector bunlde $T M \oplus T^{*} M$ has been variously called the "big tangent bundle," by Izu Vaisman, and the "Pontryagin bundle," by Hiroaki Yoshimura and Jerrold E. Marsden in [55], "because of its fundamental role in the [geometric interpretation of] Pontryagin's maximum principle" in control theory. They point out that the Whitney sum, $T Q \oplus T^{*} Q$, was first investigated in Lagrangian mechanics by Ray Skinner and Raymond Rusk [47], who themselves refer to earlier work by Mark Gotay and James N. Nester.
} 
In what follows I shall try to describe how and when a non-skewsymmetric bracket on a Courant algebroid was determined, and why it was eventually preferred in many circumstances to the skewsymmetric version, and I shall explain why it is now called the Courant-Dorfman bracket, or sometimes just the Dorfman bracket.

Alan Weinstein's first article on Dirac structures, with Ted Courant ${ }^{8}$, his doctoral student at the time, "Beyond Poisson structures", appeared in 1988 in the proceedings of the "Journées lyonnaises de la Société Mathématique de France (26-30 mai 1986) dédiées à A. Lichnerowicz" [4]. Motivated by the work of Robert Littlejohn ${ }^{9}$ on the Hamiltonian theory of guiding center motion [26, 27, 28], their idea was to define Poisson brackets on subalgebras of the algebra of smooth functions on a smooth manifold, such as the constrained brackets of Dirac, or the brackets of functions constant on the characteristic foliation of a degenerate 2-form. They succeeded in interpreting geometrically Dirac's brackets as they appeared in his Lectures on Quantum Mechanics [5] by setting up a framework unifying Poisson and presymplectic structures. What generalizes both bivector fields and differential 2-forms on a manifold $M$, or rather their graphs, from $T^{*} M$ to $T M$ for bivectors, and from $T M$ to $T^{*} M$ for forms, are subbundles of $T M \oplus T^{*} M$ that are maximally isotropic (with respect to the canonical symmetric, fiberwise bilinear form), which they called "Dirac bundles" 10 .

The novelty was in the introduction of the direct sum of the tangent and cotangent bundles. The difficulty lay in the definition of a 3-tensor on the subbundle whose vanishing was the desired integrability condition that would reduce to $[\pi, \pi]=0$, i.e., the vanishing of the SchoutenNijenhuis bracket of $\pi$, when the subbundle is the graph of a bivector $\pi$, and would reduce to $\mathrm{d} \omega=0$, i.e., $\omega$ is closed, when the subbundle is the graph of a 2 -form $\omega$. They solved this problem by introducing a trilinear map $T$ on sections of $T M \oplus T^{*} M$ (see [4, p. 44-45]). In the current literature, "integrable Dirac subbundles" are often simply called "Dirac bundles", and they are said to define a "Dirac structure" on the base manifold.

Two years later, an important, additional novelty appeared in Ted Courant's thesis, "Dirac manifolds", which was published in the Transactions of the American Mathematical Society in 1990 [3]. He succeeded in defining a skewsymmetric bracket on $T M \oplus T^{*} M$, later called "the Courant bracket", and he defined a Dirac subbundle to be "integrable" if its space of sections is closed under this bracket, in which case it is a Lie algebroid. For sections $(X, \xi)$ and $(Y, \eta)$ of $T M \oplus T^{*} M$, the Courant bracket is

$$
[(X, \xi),(Y, \eta)]=\left([X, Y], \mathcal{L}_{X} \eta-\mathcal{L}_{Y} \xi-\frac{1}{2} \mathrm{~d}\left(i_{X} \eta-i_{Y} \xi\right)\right) .
$$

Courant immediately stated, "In general, this is not a Lie-algebra bracket", and this fact prompted some of the later developments described below. The 3-tensor $T$ of the joint paper [4] could be expressed simply in terms of this new skewsymmetric bracket, and Courant proved that the integrability of a Dirac subbundle is equivalent to the vanishing of $T$, thus showing that his definition of "integrability" was equivalent to the one given in [4]. Therefore $T$ appeared as the defect in the Jacobi identity for the skewsymmetric Courant bracket, but this was not yet stated explicitly.

There arose the general question of how to define a suitable notion of the double of a Lie bialgebroid. In 1997, Zhang-Ju Liu, Alan Weinstein and Ping Xu proposed a solution to this problem in their article, "Manin triples for Lie bialgebroids" [29], by defining a skewsymmetric bracket on the space of sections of $A \oplus A^{*}$, where $\left(A, A^{*}\right)$ is a Lie bialgebroid, that generalizes the Courant bracket of $T M \oplus T^{*} M$. They further introduced a general notion of "Courant algebroids" (with a non-degenerate, symmetric, fiberwise bilinear form and a skewsymmetric

\footnotetext{
${ }^{8}$ Ted Courant is a grandson of the mathematician Richard Courant (1888-1972).

${ }^{9}$ This motivation was recalled in an e-mail message I received from Alan Weinstein (July 23, 2012).

${ }^{10}$ See Yoshimura and Marsden [55] for elements of the history of the introduction of Dirac structures on vector spaces and on manifolds, and of their use in the study of degenerate Lagrangian systems and in control theory.
} 
bracket), abstracted from the structure of the doubles of Lie bialgebroids. They wrote in their introduction: "We found that if the bracket on a Courant algebroid is modified by the addition of a symmetric term, many of the anomalies for the resulting asymmetric [i.e., non-skewsymmetric] bracket become zero," and they asked, "What is the geometric meaning of such asymmetric brackets?" In a remark [29, p. 554], they introduced a non-skewsymmetric bracket that they called "a twisted bracket", and listed three of its properties. It is easy to show that its skewsymmetrization is indeed the Courant bracket. They wrote, "It would be nice to interpret equation (i) [the Jacobi identity with a non-zero right-hand-side] in terms of this twisted bracket. The geometric meaning of this twisted bracket remains a mystery to us." All that was missing was the interpretation of this bracket as a Loday bracket satisfying the Jacobi identity in Leibniz form and, in fact, Ševera and Weinstein wrote later, "It was observed [in 1998] by KosmannSchwarzbach, Xu, and Ševera (all unpublished) that the non-skewsymmetric version of the bracket satisfied the Jacobi identity written in Leibniz form" ([46, p. 146], also see [14, p. 527]). Jean-Louis Loday ${ }^{11}$ had introduced the concept of what he called "Leibniz algebras" in 1993 [30], but they have since been called "Loday algebras". They are vector spaces equipped with a nonskewsymmetric version of the Lie brackets of Lie algebras that satisfies the following form of the Jacobi identity,

$$
[x,[y, z]]=[[x, y], z]+[y,[x, z]]
$$

which states that, for each element $x$, the adjoint map $[x, \cdot]$ is a derivation of the bracket, recalling the Leibniz rule for the derivative of the product of two functions, hence the name "Leibniz algebra".

In her e-mail message to Alan Weinstein of September 18, 1998, Kosmann-Schwarzbach demonstrated that the Courant bracket on $T M \oplus T^{*} M$ is a "derived bracket" 12 . This proof was published only much later in her "Derived brackets" paper [15], where the non-skewsymmetric bracket is obtained in a natural way as the derived bracket of the commutator of endomorphims of the space of differential forms by the de Rham differential, where vectors act by interior multiplication and 1-forms act by exterior multiplication. Then the Courant bracket appears as the skew-symmetrization of this derived bracket.

In Kosmann-Schwarzbach's notes on her conversation with Pavol Ševera at IHÉS on December 21, 1998, it is stated that together they had verified that the Dirac structures of Courant and Weinstein coincide with those of Dorfman as defined in 1987 in [7]. Later, in her book [6], Dorfman described Dirac structures and applied them to the theory of integrable equations. She wrote in the introduction, "Objects called Dirac structures were introduced by Dorfman [7] as natural algebraic analogues of finite-dimensional structures first introduced by Courant and Weinstein", and she referred to their work. Her citation, in both her article and her book, of the work of Courant and Weinstein as a Berkeley preprint dated 1986 was probably generous, because her own work on "the algebraic framework" for Dirac structures was, in fact, independent of theirs. We remark that, although she had finally visited the West to participate in the workshop on "The Geometry of Hamiltonian Systems" in Berkeley in June 1989, she had not seen their article in published form, in the 1988 volume of the "Séminaire Sud-Rhodanien de Géométrie" [4], before she completed her book.

It was Dmitry Roytenberg, another of Alan Weinstein's doctoral students, who made further progress in the theory of Courant algebroids in his thesis [41]. He introduced the nonskewsymmetric bracket, gave a new definition of Courant algebroids, containing five axioms,

\footnotetext{
${ }^{11}$ Jean-Louis Loday (born 1946) died in an accident at sea off the coast of Brittany in June 2012.

${ }^{12}$ I defined the concept of derived bracket in 1995, inspired by unpublished notes that Jean-Louis Koszul had sent me, and I published the definition and properties of derived brackets with applications to Poisson geometry in the Annales de l'Institut Fourier in 1996 [17]. This concept was also known to Ted Voronov, who later developed the theory of higher derived brackets and their relation to $L_{\infty}$-algebras.
} 
in terms of this bracket, and he proved the equivalence of the old and the new definitions. These axioms can also be found in the first of Ševera's e-mail letters to Weinstein of 1998 [45]. In 2001, Ševera and Weinstein published this definition of Courant algebroids in [46], and Roytenberg finally published it in 2002 in his "Graded symplectic supermanifolds and Courant algebroids" [43]. In his thesis, Roytenberg introduced a new interpretation of the double of a Lie bialgebroid, as a homological ${ }^{13}$ Hamiltonian vector field on an even symplectic supermanifold, thus extending the definition of a Lie algebroid structure as a homological vector field, due to Arkady Vaintrob [49]. Combining the "cotangent philosophy" of Mackenzie with what he called "Kosmann-Schwarzbach's picture of a Lie bialgebra"14, he recovered the non-skewsymmetric bracket on $A \oplus A^{*}$ as the restriction of a derived bracket of the canonical symplectic structure on a graded version of the cotangent bundle, $T^{*}(A) \approx T^{*}\left(A^{*}\right)$.

In 2002, Kyousuke Uchino showed that three of the axioms and one defining condition implied the other two axioms [48]. A year later, Janusz Grabowski and Giuseppe Marmo proposed a definition of the more general Courant-Jacobi algebroids that required only four axioms [9]. Then in 2005, Kosmann-Schwarzbach proved that three of Roytenberg's axioms for Courant algebroids imply the other two [20]. One of these three axioms is the Jacobi identity in Leibniz form for the non-skewsymmetric bracket. The other two are expressed, as in Roytenberg's thesis, in a form that clearly shows that Courant algebroids are a vector-bundle version of the Lie algebras with an invariant symmetric bilinear form ("quadratic Lie algebras") 15 .

For the case of a Courant algebroid which is the double of a Lie bialgebroid or, more generally, of a quasi-Lie bialgebroid, or of a proto-bialgebroid, $\left(A, A^{*}\right)$, the formula for the CourantDorfman bracket is a straightforward generalization of the case of the generalized tangent bundles, using the "big bracket" 16 on the algebra of functions on $T^{*}[2] A[1]$ as defined by Roytenberg, first in [41], then in his publication [44] in 2002, and the derived bracket formula. In particular, for the non-skewsymmetric bracket on $T M \oplus T^{*} M$, the expression of the derived bracket,

$$
[X+\xi, Y+\eta]=\{\{X+\xi, \mu\}, Y+\eta\}=[X, Y]+\mathcal{L}_{X} \eta-i_{Y} \mathrm{~d} \xi
$$

for $X, Y \in \Gamma(T M), \xi, \eta \in \Gamma\left(T^{*} M\right)$, where $\mu$ is the Lie bracket of vector fields seen as a function on the supermanifold $T^{*}[2] T M[1]$, coincides with the expression considered by Dorfman, in the context of complexes over Lie algebras, in [7, p. 242], and in her Theorem 2.1 in [6],

$$
[X+\xi, Y+\eta]=[X, Y]+i_{X} \mathrm{~d} \eta-i_{Y} \mathrm{~d} \xi+\mathrm{d}\langle X, \eta\rangle
$$

in order to characterize Dirac structures. Whence, by extension, the name "Dorfman bracket" that is now given to the non-skewsymmetric bracket on any Courant algebroid.

In the more general case of an arbitrary Courant algebroid, it was Roytenberg who proved in 2002 that the non-skewsymmetric Courant bracket is a derived bracket [43]. To this end, he extended his own work on Lie bialgebroids in his thesis and in [44], defining a graded Poisson bracket on the "minimal symplectic realization" of the bundle, based on a construction that had

\footnotetext{
${ }^{13} \mathrm{~A}$ vector field $X$ on a supermanifold is homological if $[X, X]=0$. It endows the manifold with the structure of a "Q-manifold".

${ }^{14}$ See footnote 16 below.

${ }^{15}$ The proof that the Leibniz rule and the morphism property of the anchor are a consequence of these three axioms followed the same lines as that of a property of Lie algebroids in our paper with Franco Magri [22]. We remark that the redundancy of some of the axioms of Courant algebroids is proved using the assumption that the symmetric bilinear form is non-degenerate. In the more general case considered in [1] and [42], where no such assumption is made, additional axioms are needed.

${ }^{16}$ The "big bracket" for vector spaces can be found in the paper by Bertram Kostant and Shlomo Sternberg [23]. It was first applied to the theory of Lie bialgebras by Pierre Lecomte and Claude Roger in 1990, and I later made extensive use of it, in [18], and in subsequent publications.
} 
been suggested by Alan Weinstein in the Spring of 1999 and later simplified by Pavol Ševera in an unpublished letter to Weinstein $[45 \text {, no. } 7]^{17}$.

Not only do Dirac structures have many applications in mechanics, as developed in the work of Jerrold Marsden (1942-2010) and many others, in the AKSZ sigma-models as was first shown by Noriaki Ikeda [13] (see [40]), and even in supergravity as in the articles of Daniel Waldram and his co-authors (see, e.g., [10]), many purely theoretical developments have taken place since 2002. Twisted Courant algebroids, also called Courant algebroids with background, were introduced. Ševera defined a cohomology class, now called "the Ševera class". In 2007, Paul Bressler defined a Pontryagin class in the generalized setting of transitive Lie algebroids, and he showed that it is an obstruction to the existence of a "Courant extension"; in addition, he related the theory of Courant algebroids to conformal field theory [1]. Then, in 2009, there appeared the article by Roytenberg where he defined and studied an algebraic analogue of Courant algebroids which he called "Courant-Dorfman algebras" [42]. Their relationship to Courant algebroids is analogous to that of Lie-Rinehart algebras to Lie algebroids ${ }^{18}$. The 2012 thesis by David Li-Bland [24] not only constitutes a new contribution to the theory of Courant algebroids, but also contains a useful list of references.

Symmetries are a fundamental feature of mathematical and physical theories. Lie's continuous groups, which are now called Lie groups, and their infinitesimal counterparts, which are now called Lie algebras, have become too restrictive a framework for geometry, algebra and mathematical physics. Lie groupoids and Lie algebroids offer a more general one, and Courant algebroids have become a new, necessary concept in this wider framework, where Drinfeld's double of a Lie bialgebra did not have an obvious analogue. Lie algebroids and Courant algebroids are, in a sense, infinitesimal objects. Lie algebroids correspond to Lie groupoids. Lie bialgebras correspond to Poisson-Lie groups. To what do Courant algebroids correspond? All I know is that Jean-Louis Loday had proposed to call these unknown objects "coquecigrues" and that, although some advances have been made recently, the search for coquecigrues ${ }^{19}$ is still on.

\section{Acknowledgements}

I wish to thank Alan Weinstein as well as Dmitry Roytenberg and Pavol Ševera for their very useful correspondence and discussions concerning their work on Courant algebroids. Thanks are also due to Ted Voronov and to the referees for their constructive remarks that were incorporated in the present version. This short history was begun as a memorandum written for Shlomo Sternberg, whom I thank for giving me the incentive to delve into some near-contemporary history.

\section{References}

[1] Bressler P., The first Pontryagin class, Compos. Math. 143 (2007), 1127-1163, math.AT/0509563.

[2] Coste A., Dazord P., Weinstein A., Groupoïdes symplectiques, in Publ. Dép. Math. Nouvelle Sér. A, Vol. 2, Univ. Claude Bernard-Lyon 1, 1987, 1-62.

[3] Courant T., Dirac manifolds, Trans. Amer. Math. Soc. 319 (1990), 631-661.

[4] Courant T., Weinstein A., Beyond Poisson structures, in Actions hamiltoniennes de groupes. Troisième théorème de Lie (Lyon, 1986), Travaux en Cours, Vol. 27, Hermann, Paris, 1988, 39-49.

\footnotetext{
${ }^{17}$ In 2012, David Li-Bland and Eckhard Meinrenken proposed a new construction of the relevant Poisson bracket. See Shlomo Sternberg's lectures at Harvard in 2012 and at the Conference in memory of Jean-Marie Souriau, Aix-en-Provence, June 2012.

18 "Lie-Rinehart algebras" is the name given by Johannes Huebschmann in 1990 to the algebraic counterpart of Lie algebroids. They are also called Lie pseudo-algebras.

${ }^{19}$ Rabelais, Gargantua, I.49. Picrochole is told by an old hag "que son royaulme luy serait rendu à la venue des cocquecigrues".
} 
[5] Dirac P.A.M., Lectures on quantum mechanics, Belfer Graduate School of Science Monographs Series, Vol. 2, Belfer Graduate School of Science, New York, 1964.

[6] Dorfman I.Ya., Dirac structures and integrability of nonlinear evolution equations, Nonlinear Science: Theory and Applications, John Wiley \& Sons Ltd., Chichester, 1993.

[7] Dorfman I.Ya., Dirac structures of integrable evolution equations, Phys. Lett. A 125 (1987), 240-246.

[8] Drinfel'd V.G., Hamiltonian structures on Lie groups, Lie bialgebras and the geometric meaning of classical Yang-Baxter equations, Soviet Math. Doklady 27 (1983), 68-71.

[9] Grabowski J., Marmo G., The graded Jacobi algebras and (co)homology, J. Phys. A: Math. Gen. 36 (2003), 161-181, math.DG/0207017.

[10] Graña M., Minasian R., Petrini M., Waldram D., T-duality, generalized geometry and non-geometric backgrounds, J. High Energy Phys. 2009 (2009), no. 4, 075, 39 pages, arXiv:0807.4527.

[11] Gualtieri M., Generalized complex geometry, Ann. of Math. (2) 174 (2011), 75-123, math.DG/0703298.

[12] Hitchin N., Generalized Calabi-Yau manifolds, Q. J. Math. 54 (2003), 281-308, math.DG/0209099.

[13] Ikeda N., Chern-Simons gauge theory coupled with BF theory, Internat. J. Modern Phys. A 18 (2003), 2689-2701, hep-th/0203043.

[14] Kinyon M.K., Weinstein A., Leibniz algebras, Courant algebroids, and multiplications on reductive homogeneous spaces, Amer. J. Math. 123 (2001), 525-550, math.DG/0006022.

[15] Kosmann-Schwarzbach Y., Derived brackets, Lett. Math. Phys. 69 (2004), 61-87, math.DG/0312524.

[16] Kosmann-Schwarzbach Y., Exact Gerstenhaber algebras and Lie bialgebroids, Acta Appl. Math. 41 (1995), $153-165$.

[17] Kosmann-Schwarzbach Y., From Poisson algebras to Gerstenhaber algebras, Ann. Inst. Fourier (Grenoble) 46 (1996), 1243-1274.

[18] Kosmann-Schwarzbach Y., Jacobian quasi-bialgebras and quasi-Poisson Lie groups, in Mathematical Aspects of Classical Field Theory (Seattle, WA, 1991), Contemp. Math., Vol. 132, Editors M. Gotay, J.E. Marsden, V. Moncrief, Amer. Math. Soc., Providence, RI, 1992, 459-489.

[19] Kosmann-Schwarzbach Y., Poisson manifolds, Lie algebroids, modular classes: a survey, SIGMA 4 (2008), 005, 30 pages, arXiv:0710.3098.

[20] Kosmann-Schwarzbach Y., Quasi, twisted, and all that ... in Poisson geometry and Lie algebroid theory, in The Breadth of Symplectic and Poisson Geometry, Festschrift in Honor of Alan Weinstein, Progr. Math., Vol. 232, Editors J.E. Marsden, T.S. Ratiu, Birkhäuser Boston, Boston, MA, 2005, 363-389, math.DG/0310359.

[21] Kosmann-Schwarzbach Y. (Editor), Siméon-Denis Poisson. Les Mathématiques au service de la science, Éditions de l'École Polytechnique, to appear.

[22] Kosmann-Schwarzbach Y., Magri F., Poisson-Nijenhuis structures, Ann. Inst. H. Poincaré Phys. Théor. 53 (1990), 35-81.

[23] Kostant B., Sternberg S., Symplectic reduction, BRS cohomology, and infinite-dimensional Clifford algebras, Ann. Physics 176 (1987), 49-113.

[24] Li-Bland D., LA-Courant algebroids and their applications, Ph.D. thesis, University of Toronto, 2012, arXiv:1204.2796.

[25] Lichnerowicz A., Les variétés de Poisson et leurs algèbres de Lie associées, J. Differential Geometry 12 (1977), 253-300.

[26] Littlejohn R.G., A guiding center Hamiltonian: a new approach, J. Math. Phys. 20 (1979), 2445-2458.

[27] Littlejohn R.G., Geometry and guiding center motion, in Fluids and Plasmas: Geometry and Dynamics (Boulder, Colo., 1983), Contemp. Math., Vol. 28, Editor J.E. Marsden, Amer. Math. Soc., Providence, RI, 1984, 151-167.

[28] Littlejohn R.G., Hamiltonian formulation of guiding center motion, Phys. Fluids 24 (1981), $1730-1749$.

[29] Liu Z.-J., Weinstein A., Xu P., Manin triples for Lie bialgebroids, J. Differential Geom. 45 (1997), 547-574, dg-ga/9508013.

[30] Loday J.-L., Une version non commutative des algèbres de Lie: les algèbres de Leibniz, Enseign. Math. (2) 39 (1993), 269-293.

[31] Mackenzie K.C.H., Double Lie algebroids and second-order geometry. I, Adv. Math. 94 (1992), $180-239$. 
[32] Mackenzie K.C.H., Double Lie algebroids and second-order geometry. II, Adv. Math. 154 (2000), 46-75, dg-ga/9712013.

[33] Mackenzie K.C.H., Drinfel'd doubles and Ehresmann doubles for Lie algebroids and Lie bialgebroids, Electron. Res. Announc. Amer. Math. Soc. 4 (1998), 74-87.

[34] Mackenzie K.C.H., Ehresmann doubles and Drinfel'd doubles for Lie algebroids and Lie bialgebroids, J. Reine Angew. Math. 658 (2011), 193-245, math.DG/0611799.

[35] Mackenzie K.C.H., General theory of Lie groupoids and Lie algebroids, London Mathematical Society Lecture Note Series, Vol. 213, Cambridge University Press, Cambridge, 2005.

[36] Mackenzie K.C.H., Xu P., Lie bialgebroids and Poisson groupoids, Duke Math. J. 73 (1994), 415-452.

[37] Mokhov O.I., Novikov S.P., Pogrebkov A.K., Irina Yakovlevna Dorfman, Russ. Math. Surv. 50 (1995), $1241-1246$.

[38] Olver P.J., Applications of Lie groups to differential equations, Graduate Texts in Mathematics, Vol. 107, Springer-Verlag, New York, 1986.

[39] Pradines J., Théorie de Lie pour les groupoïdes différentiables. Calcul différenetiel dans la catégorie des groupoïdes infinitésimaux, C. R. Acad. Sci. Paris Sér. A-B 264 (1967), A245-A248.

[40] Roytenberg D., AKSZ-BV formalism and Courant algebroid-induced topological field theories, Lett. Math. Phys. 79 (2007), 143-159, hep-th/0608150.

[41] Roytenberg D., Courant algebroids, derived brackets and even symplectic supermanifolds, Ph.D. thesis, University of California, Berkeley, 1999, math.DG/9910078.

[42] Roytenberg D., Courant-Dorfman algebras and their cohomology, Lett. Math. Phys. 90 (2009), 311-351, arXiv:0902.4862.

[43] Roytenberg D., On the structure of graded symplectic supermanifolds and Courant algebroids, in Quantization, Poisson Brackets and Beyond (Manchester, 2001), Contemp. Math., Vol. 315, Editor T. Voronov, Amer. Math. Soc., Providence, RI, 2002, 169-185, math.SG/0203110.

[44] Roytenberg D., Quasi-Lie bialgebroids and twisted Poisson manifolds, Lett. Math. Phys. 61 (2002), 123-137, math.QA/0112152.

[45] Ševera P., Letters to Alan Weinstein written between 1998 and 2000 (no. 1, 1998, no. 7, 2000), available at http://sophia.dtp.fmph.uniba.sk/ severa/letters/.

[46] Ševera P., Weinstein A., Poisson geometry with a 3-form background, Progr. Theoret. Phys. Suppl. 144 (2001), 145-154, math.SG/0107133.

[47] Skinner R., Rusk R., Generalized Hamiltonian dynamics. I. Formulation on $T^{*} Q \oplus T Q$, J. Math. Phys. 24 (1983), 2589-2594.

[48] Uchino K., Remarks on the definition of a Courant algebroid, Lett. Math. Phys. 60 (2002), 171-175, math.DG/0204010.

[49] Vaintrob A.Yu., Lie algebroids and homological vector fields, Russ. Math. Surv. 52 (1997), 428-429.

[50] Voronov T., Graded manifolds and Drinfeld doubles for Lie bialgebroids, in Quantization, Poisson Brackets and Beyond (Manchester, 2001), Contemp. Math., Vol. 315, Editor T. Voronov, Amer. Math. Soc., Providence, RI, 2002, 131-168, math.DG/0105237.

[51] Voronov T., Q-manifolds and Mackenzie theory, Comm. Math. Phys. 315 (2012), 279-310, arXiv:1206.3622.

[52] Weinstein A., Coisotropic calculus and Poisson groupoids, J. Math. Soc. Japan 40 (1988), 705-727.

[53] Weinstein A., Poisson geometry, Differential Geom. Appl. 9 (1998), 213-238.

[54] Weinstein A., Sophus Lie and symplectic geometry, Exposition. Math. 1 (1983), 95-96.

[55] Yoshimura H., Marsden J.E., Dirac structures in Lagrangian mechanics. I. Implicit Lagrangian systems, J. Geom. Phys. 57 (2006), 133-156. 